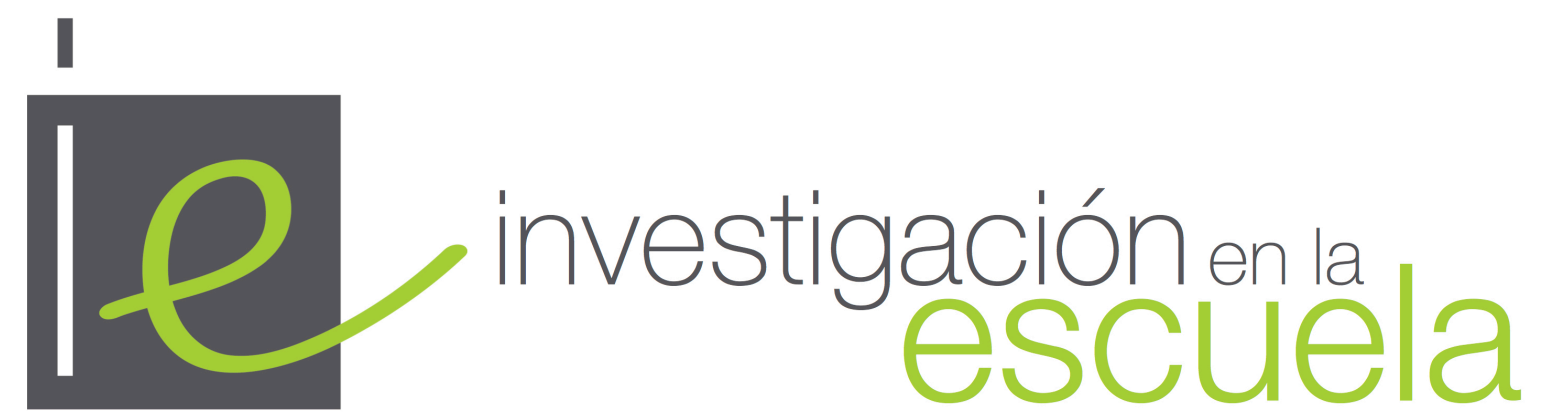

Revista internacional de investigación e innovación educativa

\title{
Las emociones en las metáforas personales de futuros profesores de Secundaria en relación con el rol del profesor y del alumnado
}

\author{
Lucía Mellado ${ }^{1}$ Juan Luis de la Montaña ${ }^{2}$, María Rosa Luengo y María Luisa \\ Bermejo $^{2}$ \\ ${ }^{1}$ UNED, ${ }^{2}$ Universidad de Extremadura \\ España
}

Citación: Mellado, L., De la Montaña, J. L., Luengo, M. R., \& Bermejo, M. L. (2017). Las emociones en las metáforas personales de futuros profesores de Secundaria en relación con el rol del profesor y del alumnado. Investigación en la Escuela, 91, 36-55. Recuperado de http://www.investigacionenelaescuela.es/articulos/R91/R91-3

Resumen: Este estudio analiza, a través de un cuestionario de preguntas abiertas, la evolución de las metáforas personales de una muestra de la especialidad de Economía del Máster Universitario en Formación del Profesorado en Educación Secundaria, durante el curso 2012/2013. Para el análisis se han considerado cuatro categorías: conductistas-transmisivas, cognitivas-constructivistas, situadas y autorreferenciadas. Al comparar las metáforas del profesor, antes y después de las prácticas de enseñanza, se produce una evolución progresiva de las metáforas conductistas-transmisivas a cognitivas-constructivistas y situadas. La mayor parte de las emociones asociadas a las metáforas fueron sociales, tanto para el profesor como para el alumnado. La mayoría de los participantes no cambiaron sus modelos didácticos, que estaban ya firmemente asentados al comienzo del Máster. Sin embargo, en algunos se produce una evolución progresiva hacia modelos más centrados en los estudiantes. 
Palabras clave: "Metáforas"; “emociones"; "formación del profesorado"; “enseñanza secundaria”; "Ciencias Económicas".

\section{Emotions in Personal Metaphors of Future Secondary Teachers about the role of the teacher and the pupils}

Abstract: Using the responses to open questions, this study analyzes the evolution of personal metaphors of a sample of prospective secondary economics teacher during their Master's degree course in Secondary Teacher Education during the 2012/2013 academic year. For the analysis, four categories have been considered: transmissive/behavioral, cognitive/constructivist, situated and selfreferential. Comparing teacher' metaphors before and after teaching practice, a progressive evolution occurs from transmissive-constructivist metaphors to cognitive-behavioral and situated metaphors. Most of the emotions associated with the metaphors were social, both for the teacher and for the pupils. The results revealed no changes in most of the participants' associated models. Instead, these appeared to be firmly set already at the beginning of the Master's course. However in some there is a progressive evolution towards more student-centered models.

Key words: "Metaphors"; "emotions"; "teacher training"; "secondary education"; "economics".

Les émotions dans les métaphores personnelles des futurs professeurs de l'enseignement secondaire sur le rôle du professeur et des élèves

Resumè: Cette étude analyse, au moyen d'un questionnaire de questions ouvertes, l'évolution des métaphores personnelles dans un échantillon d'étudiants de la spécialité d'Économie du Master de Formation de Professeurs d'enseignement secondaire, pendant l'année académique 2012/2013. L'analyse se base sur quatre catégories: comportementaliste-transmissive, cognitive-constructiviste, située et autoréférenciée. En comparant les métaphores du professeur, avant et après le stage pratique d'enseignement, nous observons une évolution progressive des métaphores comportementaliste-transmissive vers les cognitive-constructive et située. La plupart des émotions associées aux métaphores ont été sociales, tant pour le professeur comme pour les élèves. Une majorité des participants n'a pas changé ses modèles didactiques, qui étaient fermement établis au début du Master. Cependant, certains participants montrent une évolution progressive vers des modèles plus centrés sur les étudiants.

Mostsclé: "Métaphores"; "émotions"; "formations de professeurs"; “enseignement secondaire"; "économie".

\section{Introducción}

La alfabetización económica de los estudiantes es fundamental para procurar a los futuros ciudadanos recursos esenciales con los que participar activamente en la construcción de sociedades modernas y democráticas (Cañas y de la Montaña, 2012). Para Molina y Travé (2014) la incorporación de las asignaturas de Economía a la educación obligatoria requiere configurar una Didáctica de la Economía, dentro de la Didáctica de las Ciencias Sociales, que fundamente la formación inicial y continua de este profesorado.

En 1985 el Consejo Nacional de Educación Económica de Estados Unidos, manifestó su preocupación por ayudar a los docentes a enseñar Economía, y destacó la formación del profesorado como elemento clave. Salemi, Siegfried, Sosin, Walstad y Watts (2001) insisten en la formación inicial como paso previo para implementar métodos de enseñanza de la Economía 
basados en el aprendizaje activo de los estudiantes. En su interés por mejorar la formación de los docentes de Economía, Salemi (2003) propone un modelo de formación en el que ofrece ideas sobre objetivos, contenidos y métodos, y en el que destaca la aportación de las prácticas de enseñanza para el profesorado en formación.

Desde la década de los noventa se han desarrollado distintas líneas de investigación en Didáctica de la Economía, siendo una de ellas el estudio de las concepciones y la práctica del profesorado de secundaria de Economía, tanto en formación como en servicio (Travé, 1998; Travé y Pozuelos, 2008). La comprensión de los factores que favorecen u obstaculizan la formación y el desarrollo profesional del profesorado es actualmente uno de los temas más relevantes de la agenda de la investigación educativa y un elemento esencial para planificar y llevar a cabo programas de formación que tengan como resultado una mejora de la enseñanza y del aprendizaje en las aulas (Estepa y Cuenca, 2007). Con la implantación de la especialidad de Economía en el Máster Universitario en Formación del Profesorado en Educación Secundaria (en adelante MFPES) se ha dado un importante paso en la formación y la identidad profesional de este profesorado.

Sin embargo, cuando los futuros profesores de Economía se incorporan al Máster, lo hacen no sólo con un bagaje de conocimientos, sino con unos valores, concepciones, roles y actitudes sobre la enseñanza, el aprendizaje y el rol del profesor, fruto de los muchos años de su propia escolaridad (Mahlios, Massengill-Shaw y Barry, 2010). Estas concepciones didácticas personales, adquiridas de forma natural y sin reflexión de sus propias experiencias como estudiantes, están profundamente arraigadas y son difíciles de cambiar. Esta impregnación hace que muchos profesores utilicen en la práctica los métodos didácticos que ellos mismos preferían como estudiantes, o simplemente enseñan de la misma forma en que fueron enseñados (Tobin, Tippins y Gallard, 1994). Por otra parte Molina y Travé (2014) constatan que en algunos aspectos del currículo, los profesores de Economía en ejercicio pueden tener un discurso dual entre lo que consideran deseable y la puesta en práctica en el aula de estos modelos. Por tanto un primer paso en la formación es que los profesores analicen sus propias concepciones y modelos didácticos, sean conscientes de los mismos y de las posibles alternativas y tengan los conocimientos y herramientas necesarias para controlar y autorregular sus propios cambios (Cañal, Travé y Pozuelos, 2011).

En este artículo presentamos una investigación longitudinal en la que analizamos, a través de las metáforas personales, la evolución de las concepciones sobre el rol del profesor y sobre el aprendizaje del alumnado, así como de las emociones, de un grupo de futuros profesores de Economía del MFPES, antes y después de las prácticas de enseñanza. El trabajo forma parte de una investigación más amplia, realizada por un equipo interdisciplinar con profesorado de distintos niveles y especialización, de la que a continuación resumimos el marco teórico, ya desarrollado en otros trabajos (Mellado, de la Montaña, Luengo y Bermejo, 2016 a y b).

\section{Las metáforas personales y las emociones del profesorado}

Cada profesor elabora un pensamiento práctico profesional idiosincrásico, a partir de su experiencia personal y de la interacción social con el entorno que le rodea. El lenguaje que utiliza el docente para hablar de sus concepciones, roles y de su actividad profesional no suele ser literal y estructurado, sino más bien simbólico y de carácter metafórico (Lakoff y Johnson, 1986).

Una metáfora es la sustitución o transposición de una idea o concepto por otro que tenga con él una cierta relación de semejanza objetiva o subjetiva para el emisor. Las metáforas se han utilizado como un recurso expresivo en la literatura, en la oratoria, en la música y en numerosas expresiones artísticas y de comunicación. Desde la psicolingüística se estudia la correlación entre 
comportamiento lingüístico y procesos psicológicos subyacentes, por lo que la metáfora sería un mecanismo heurístico que se manifiesta lingüísticamente.

Las metáforas, por tanto, no son sólo un recurso expresivo, sino que tienen un significado más profundo ya que constituyen un mecanismo esencial de la mente, que estructura gran parte del sistema conceptual por medio de relaciones metafóricas y son un principio fundamental del pensamiento y la acción (Lakoff y Johnson, 1986). Las metáforas son como lentes, pantallas o filtros de lo que somos y de cómo nos vemos en determinada situación (Saban, 2010).

En las Ciencias Económicas el estudio de las metáforas es un campo de investigación aceptado (Herrera-Soler y White, 2012) y forma parte de las teorías económicas (Fukuda, 2009), de la teoría de las organizaciones (Morgan, 1980), de las finanzas y contabilidad (Walters y Young, 2008), del mercado (Teichert, von Wartburg y Braterman, 2006), de lo comercial (Madhavaram y McDonald, 2010), de los empresarios (Dodd, 2012), de los recursos humanos (McCorkle y Gayle, 2003) y de las concepciones de estudiantes de Máster de Dirección y Administración de EmpresasMBA (Mellado, Parte y Garvey, 2016).

Para el profesorado las metáforas son un medio para establecer puentes entre las concepciones y la narrativa que describe la vida del aula (Ben-Peretz, Mendelson y Kron, 2003), ya que permiten averiguar los referentes implícitos que sustentan las concepciones y dan una visión holística de la vida del aula. Las metáforas pueden crear realidades sociales y convertirse en guía para las acciones futuras que se ajustarán a la metáfora: "las metáforas pueden ser profecías que se cumplen" (Lakoff y Johnson, 1986, p. 198). Numerosos trabajos han mostrado que los profesores realizan cambios en sus concepciones y en su práctica del aula cuando construyen nuevas metáforas compatibles con tales cambios (Martínez, Sauleda y Huber, 2001; Tobin et al., 1994).

Los profesores construyen sus metáforas a partir de sus antecedentes escolares y de su propia experiencia personal (Ben-Peretz et al., 2003), por lo que éstas tienen un fuerte componente afectivo. Las metáforas sirven de puente para unir el mundo cognitivo y afectivo y pueden ayudar a los profesores a tomar conciencia de sus sentimientos y emociones (Lakoff y Johnson, 1986; Mellado, Luengo, de la Montaña y Bermejo, 2015; Rebollo, Jiménez, Sabuco y Vega, 2013). En la enseñanza hay factores emocionales que influyen en las acciones y en la toma de decisiones de los profesores (Damasio, 2010). Las emociones son muy importantes para todos los profesores, pero especialmente para los profesores en formación, ya que las primeras experiencias de enseñanza durante el Practicum pueden ser traumáticas para los futuros profesores, que en esta etapa son especialmente vulnerables (Mellado et al., 2014). Estas emociones negativas pueden fijar estrategias conductistas, dirigidas hacia el control y la supervivencia, que son muy resistentes al cambio en el futuro.

Aunque desde la década de los 90 contamos con importantes estudios sobre las metáforas de los profesores, coincidimos con Shaw, Barry y Mahlios (2008) en que las metáforas deben seguir siendo objeto de investigación en aspectos menos tratados, como su relación con las emociones o su incidencia en el cambio de los modelos didácticos.

\section{Objetivos de la investigación}

Los objetivos de la investigación son los siguientes:

a) Clasificar las metáforas personales de la muestra dentro de las categorías conductistatransmisiva, cognitiva-constructivista, situadas-sociales y auto-referenciadas, y determinar su evolución, antes y después de las prácticas de enseñanza.

b) Determinar la evolución de los modelos didácticos asociados a las metáforas. 
c) Clasificar las metáforas personales relacionadas con las emociones en las cuatro categorías así como determinar su evolución antes y después de las prácticas de enseñanza.

d) Analizar las emociones reflejadas en las metáforas.

\section{Metodología}

La muestra la componen los futuros profesores de secundaria que realizaron el MFPES en la especialidad de Economía, en la Universidad de Extremadura durante el curso 2012-2013. Estos profesores han cursado antes estudios universitarios centrados exclusivamente en el conocimiento del contenido de una materia específica y en el MFPES es donde por primera vez cursan materias de psicología, pedagogía y didáctica de la materia específica, y en el segundo semestre realizan prácticas de enseñanza en Centros de Educación Secundaria durante tres meses.

Durante el desarrollo del Máster se les informó sobre los objetivos del estudio y la voluntariedad de participar en el mismo. El pretest se realizó antes de las prácticas de enseñanza en los Centros de Secundaria y el postest después de la realización de las mismas. Hemos identificado por el código anónimo personal a los 14 participantes que realizaron tanto el pretest como el postest, los cuales constituyen la muestra de este estudio (véase tabla 1).

Tabla 1

Características de la muestra participante en el estudio

\begin{tabular}{ccccc}
\hline & Sexo & Edad & Licenciatura & Experiencia docente \\
\hline E1 & H & $21-25$ & Economía & No \\
E2 & M & $21-25$ & Economía & No \\
E3 & M & $26-30$ & Derecho & No \\
E4 & M & $26-30$ & Economía & No \\
E5 & M & 35 & Economía & Si \\
E6 & M & $21-25$ & LADE & No \\
E7 & M & $21-25$ & LADE & No \\
E8 & M & $21-25$ & Economía & No \\
E9 & M & $21-25$ & LADE & No \\
E10 & M & $21-25$ & Economía & No \\
E11 & M & $21-25$ & Turismo & No \\
E12 & $M$ & $21-25$ & LADE & No \\
E13 & M & $31-35$ & Turismo & Si \\
E14 & H & $26-30$ & LADE & No \\
\hline
\end{tabular}

Como puede observarse en la tabla 1 , hay 12 mujeres (85.7\%) y 2 hombres (14.3\%). Como en otras carreras de sociales en la muestra hay predominio de mujeres, lo que refleja la feminización de la profesión y quizás también la relación entre la elección de la carrera y los estereotipos de género asociados a la profesión (Gutiérrez y Luengo, 2003). En cuanto a la edad, el $64.3 \%$ tienen entre 21 y 25 años, el $21.4 \%$ entre 26 y 30, y el $14.3 \% 31$ o más años. Como la investigación se realizó durante la etapa de transición al EEES, los participantes en el Máster tenían la titulación de Licenciado, ya que todavía no existían egresados de los títulos de Grado previstos 
en el EEES: la muestra la componen licenciados en Economía (42.8\%), Administración de Empresas-LADE (35.7\%), Turismo (14.3\%) y Derecho (7.2\%). Finalmente 2 participantes (el $14.3 \%$ ) indican que tienen experiencia docente y 12 que no la tienen (el 85.7\%).

El procedimiento de recogida de datos ha sido un cuestionario anónimo en el que además del sexo, la edad y los antecedentes académicos se hacen preguntas abiertas sobre sus propias metáforas como profesores y las metáforas con las que identifican al alumnado, así como las razones que les llevan a identificarse con esas metáforas. Para elaborar el cuestionario se tomaron como referencia las investigaciones previas de Leavy, McSorley y Boté (2007), de Martínez et al. (2001) y nuestros propios estudios en los que fue validado el cuestionario abierto (Mellado et al., 2015). Finalmente el cuestionario incluye también la realización de dibujos sobre las metáforas, aspecto validado en anteriores estudios con profesorado en formación (Buaraphan, 2011; Mellado, de la Montaña, Luengo y Bermejo, 2016a). Para Markic y Eilks (2015) los dibujos de los profesores son como espejos de su identidad profesional y son muy útiles para determinar si una clase está centrada en el profesor y el contenido o en los alumnos y el aprendizaje. Antes de pasar el cuestionario se les informó sobre el significado y la importancia de las metáforas en educación, pero no se les dio ningún ejemplo de metáforas personales para no condicionar sus respuestas.

En nuestro estudio adaptamos las cuatro categorías de Leavy et al. (2007): la conductistatransmisiva: el alumno es un aprendiz pasivo, el profesor un transmisor de información y la motivación depende de refuerzos externos, y tiene además la característica de ser una clase centrada en el profesor y en el contenido; la cognitiva-constructivista: al alumno se le considera un agente activo en el proceso de construcción de su propio conocimiento y al profesor como un facilitador del proceso; la situada o de aprendizaje social: el aprendizaje está centrado en el contexto social y la motivación surge del compromiso con la comunidad educativa; esta categoría se parece a la cognitiva-constructivista porque está centrada en el alumno y el aprendizaje, pero se diferencia en que es un aprendizaje más socializado y colaborativo donde se destaca el trabajo en conjunto; la autorreferenciada: son metáforas de difícil clasificación y tienen un componente relacionado con el yo personal, cuyo significado es necesario analizar en cada caso. Muchas metáforas de animales son autorreferenciadas y suelen tener una carga emocional.

Con respecto a las emociones las clasificamos en primarias o sociales y en positivas, negativas o neutras. Todas las emociones analizadas han sido reconocidas como tales por varios autores (Borrachero, Brígido, Mellado, Costillo y Mellado, 2014; Damasio, 2010).

Se realizó un análisis descriptivo de frecuencias en cada grupo y categoría y se discutieron las razones por las que se identifican con las metáforas, contrastando los resultados con anteriores estudios, así como entre las metáforas escritas y los dibujos que las representaban. Finalizado el análisis del pretest, y antes de las prácticas de enseñanza, se realizó una sesión con el grupo en el que se les expusieron en power point las metáforas y los dibujos que las representaban y se discutieron sus significados, sin dar una valoración positiva o negativa a cada una de las metáforas. La recogida de datos del postest la realizaron después de las prácticas de enseñanza, en una de las sesiones de tutoría sobre las prácticas.

Para el análisis de la evolución de los modelos didácticos se han tomado como referencia la hipótesis de progresión de Porlán y Rivero (1998) y Carroza y Travé (2012). La evolución de los modelos transmisivos-conductistas a los constructivistas-cognitivos, y de éstos a los situadossociales, se ha considerado una progresión, y lo contrario una regresión, ya que, como desde el National Research Council (1996) de EEUU se indica, las orientaciones centradas en los estudiantes son un indicador de la implementación de estrategias didácticas de indagación e innovación en el aula. 


\section{Discusión de los resultados}

Al ser una muestra elegida por conveniencia, los resultados no pretenden ser generalizables, sino más bien, desde una perspectiva interpretativa, buscamos los significados de las metáforas individuales de los participantes.

\section{Metáforas generales}

En la figura 1 se comparan los porcentajes de las metáforas sobre el profesor, en las cuatro categorías de estudio, antes (pretest) y después de las prácticas de enseñanza (postest). Antes de las prácticas de enseñanza el mayor porcentaje de metáforas para el profesorado son las transmisivasconductistas, seguidas de las cognitivas-constructivistas. Después de las prácticas, el mayor porcentaje de metáforas corresponde a las categorías cognitivas-constructivistas y situadas, destacando la fuerte disminución de las transmisivas-conductistas y el gran aumento de las situadas-sociales.

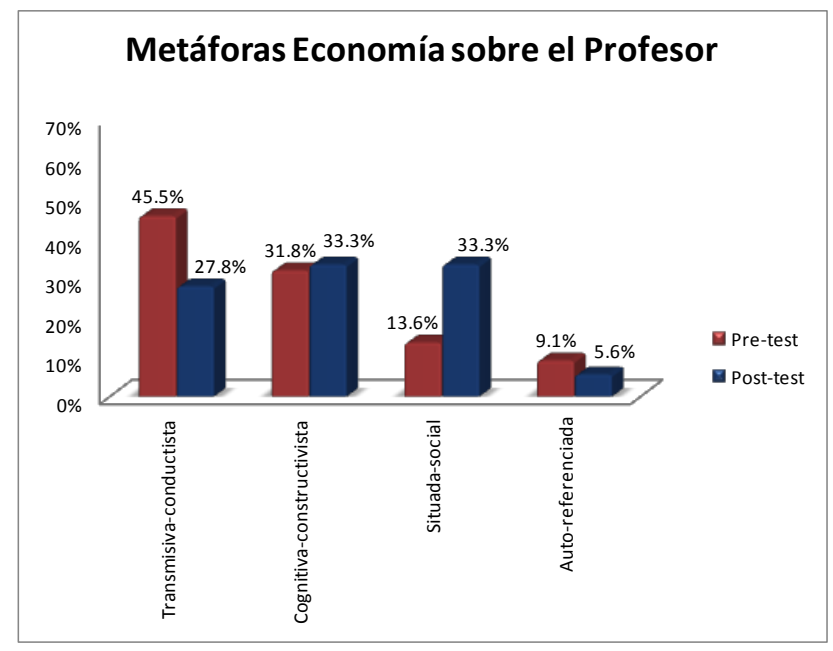

Figura 1. Evolución de las metáforas para el profesor, antes y después de las prácticas de enseñanza

Estas metáforas han sido analizadas más ampliamente en otro trabajo (Mellado et al., 2016a). Ejemplos de metáforas transmisivas-conductistas para el profesor son: la máquina, la mirada asesina, el pozo de sabiduría, el libro abierto, el tren, el líder o el padre. Entre las cognitivasconstructivistas están: el sembrador, la mano, el amigo, los rayos del sol, el perro guía, entre otras. Entre las situadas-sociales destacamos: el camino a seguir, el director de orquesta, la luz que ilumina o que guía, el guía de la escalada. Entre las autorreferenciadas se encuentran algunas de animales como el búho sabio o el cordero, entre otras.

En la figura 2 se comparan los porcentajes de las metáforas sobre el alumnado, en las cuatro categorías de estudio, antes (pretest) y después de las prácticas de enseñanza (postest). Antes de las prácticas el mayor porcentaje de metáforas para el alumnado son las autorreferenciadas seguidas de las cognitivas-constructivistas y de las transmisivas-conductistas. Después de las prácticas se produce un aumento de las metáforas transmisivas-conductistas y cognitivas-constructivistas y una fuerte disminución de las autorreferenciadas. El mayor número de metáforas autorreferenciadas antes de las prácticas puede ser indicativo de una indefinición de los modelos asociados a las 
metáforas del alumnado, que después de las prácticas se van definiendo, aunque en este caso hay poca coherencia con las categorías mostradas para el profesor.

Entre las metáforas transmisivas-conductistas para el alumnado destacamos: la esponja, el demonio, el libro cerrado, marionetas, ovejas, hormigas, pilas o carne de cañón, entre otras. Ejemplos de metáforas cognitivas-constructivistas son: semillas, plantas, frutos, seres vivos, mariposas que se transforman o construcción de sueños. Para las autorrefenciadas también para el alumnado predominan las metáforas de animales, sobre todo en el pretest: tortugas, ovejas, caracol, zorros o linces, entre otras.

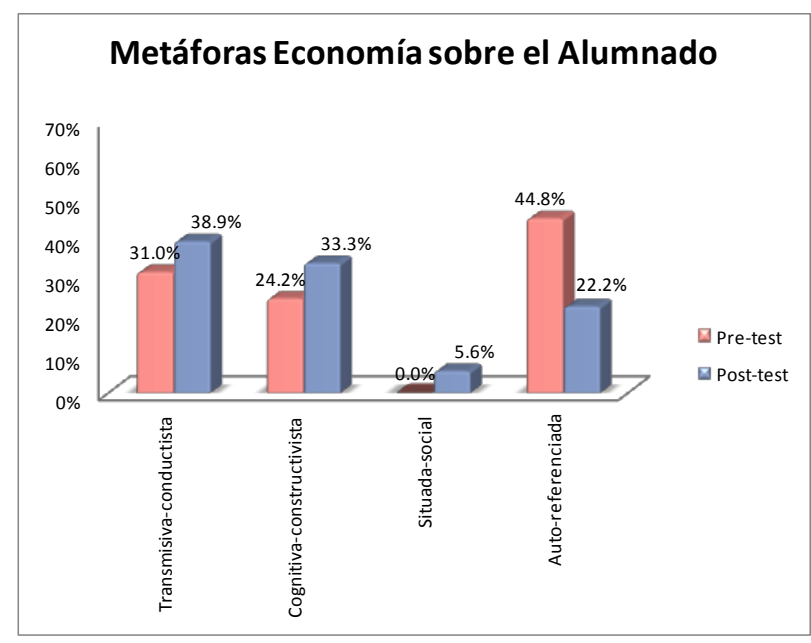

Figura 2. Evolución de las metáforas para el alumnado, antes y después de las prácticas de enseñanza

Basándonos en las metáforas dominantes, hemos determinado la evolución de los modelos didácticos de cada participante, tanto para el profesorado como para el alumnado (veáse tabla 2).

\section{Tabla 2}

Cambios en las metáforas del profesor y del alumnado, antes y después de las Prácticas de $(\mathrm{T}=$ Transmisiva-conductista; $\mathrm{C}=$ Cognitiva-Constructivista; $\mathrm{S}=$ Situada-social; $\mathrm{A}$ = Auto-referenciada)

\begin{tabular}{ccccc}
\hline $\begin{array}{c}\text { Participantes } \\
\text { de Economía }\end{array}$ & $\begin{array}{c}\text { Evolución } \\
\text { metáforas } \\
\text { profesor del } \\
\text { Pre al postest }\end{array}$ & $\begin{array}{c}\text { Evolución } \\
\text { metáforas } \\
\text { alumnado del } \\
\text { Pre al postest }\end{array}$ & $\begin{array}{c}\text { Cambios en las } \\
\text { metáforas del } \\
\text { Profesor }\end{array}$ & $\begin{array}{c}\text { Cambios en las } \\
\text { metáforas del } \\
\text { Alumnado }\end{array}$ \\
\hline E1 & T --- T & T --- T & No cambian & No cambian \\
E2 & T --- T & T --- T & No cambian & No cambian \\
E3 & T --- T & T --- T & No cambian & No cambian \\
E4 & T --- T & A --- A & No cambian & No cambian \\
E5 & C --- C & C --- C & No cambian & No cambian \\
E6 & C --- C & C --- C & No cambian & No cambian \\
E7 & S --- S & A --- A & No cambian & No cambian \\
E8 & S --- S & C --- C & No cambian & No cambian \\
E9 & S --- S & C T--- T & No cambian & Regresión \\
E10 & T --- S & A --- A & Progresión & No cambian \\
E11 & T --- CS & A --- A & Progresión & No cambian
\end{tabular}


Tabla 2 (Cont.)

Cambios en las metáforas del profesor y del alumnado, antes y después de las Prácticas de $(\mathrm{T}=$ Transmisiva-conductista; $\mathrm{C}=$ Cognitiva-Constructivista; $\mathrm{S}=$ Situada-social; $\mathrm{A}=$ Auto-referenciada)

\begin{tabular}{ccccc}
\hline $\begin{array}{c}\text { Participantes } \\
\text { de }\end{array}$ & $\begin{array}{c}\text { Evolución } \\
\text { metáforas } \\
\text { profesor } \\
\text { del Pre al } \\
\text { postest }\end{array}$ & $\begin{array}{c}\text { Evolución } \\
\text { metáforas } \\
\text { alumnado del } \\
\text { Pre al postest }\end{array}$ & $\begin{array}{c}\text { Cambios en las } \\
\text { metáforas del } \\
\text { Profesor }\end{array}$ & $\begin{array}{c}\text { Cambios en las } \\
\text { metáforas del } \\
\text { Alumnado }\end{array}$ \\
\hline E12 & T --- S & C --- C & Progresión & No cambian \\
E13 & TC --- C & T --- C & Progresión & Progresión \\
E14 & C --- S & C --- S & Progresión & Progresión \\
\hline
\end{tabular}

En las metáforas sobre el profesor (columna 4 de la tabla 2) no se producen cambios en 9 de los participantes (el 64.29\%), pero en 5 de ellos se produce una progresión de los modelos del profesor $(35.71 \%)$, no habiendo participantes que sufran regresión en sus metáforas sobre el profesor. En las metáforas sobre el alumnado (columna 5 de la tabla 2) no se producen cambios en 11 de los participantes (el 78.57\%), en 2 de ellos se produce una progresión (14.29\%) y en uno de ellos una regresión $(7.14 \%)$.

\section{Metáforas relacionadas con las emociones}

En la figura 3 se indican los porcentajes de estas metáforas para el rol del profesor en las cuatro categorías de estudio. Si en la metáforas generales el mayor porcentaje antes de las prácticas correspondía a la categoría conductista/transmisiva, en las emocionales el mayor porcentaje antes de las prácticas corresponde a la categoría cognitiva/constructivista. Después de las prácticas aumentan las metáforas en las categorías cognitiva/constructivista y situada-social y disminuyen en las conductista/transmisiva y autorreferenciada.

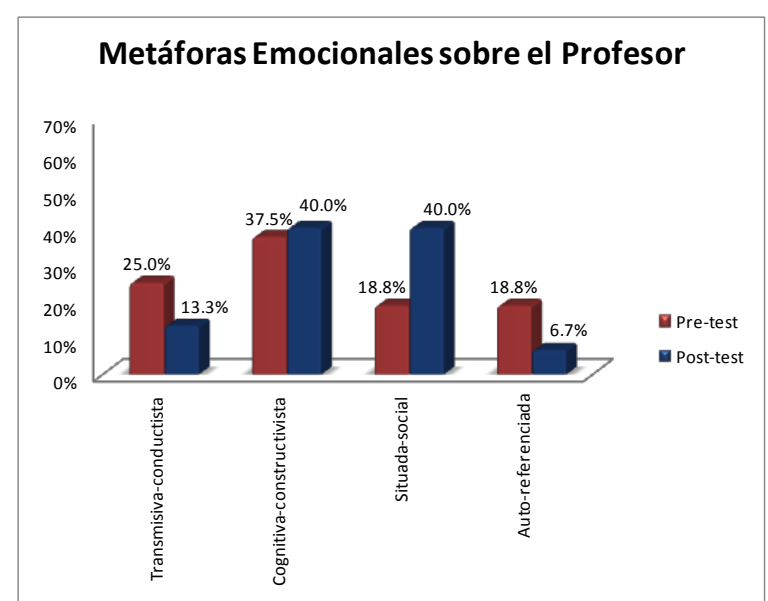

Figura 3. Evolución de las metáforas emocionales para el profesor, antes y después de las prácticas 
Para el alumnado (véase figura 4) antes de las prácticas el mayor porcentaje de metáforas emocionales corresponde las categorías cognitiva/constructivista y autorreferenciada. Después de las prácticas aumentan los porcentajes de metáforas emocionales en las categorías conductista/transmisiva y situada-social y disminuyen en las otras dos.

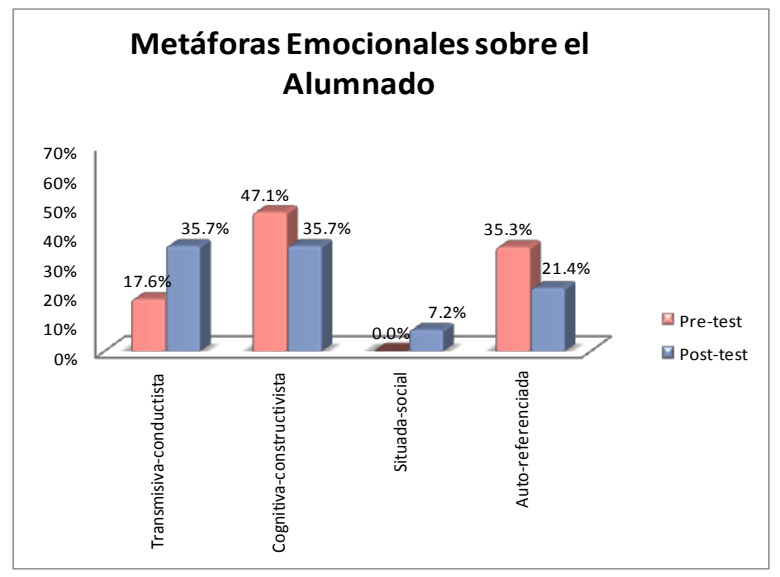

Figura 4. Evolución de las metáforas emocionales para el alumnado, antes y después de las prácticas

A continuación mostramos algunas metáforas con significado emocional, basándonos en los dibujos y en las razones que los participantes han dado para identificarse con estas metáforas.

a) Metáforas que reflejan emociones primarias positivas. Entre las metáforas que identificamos con la felicidad para el profesor en el pretest está la del sol que da luz y calor. No se han identificado metáforas con emociones primarias para el alumnado.

b) Metáforas que reflejan emociones sociales positivas. Son las más numerosas en nuestro estudio. Entusiasmo: La metáfora del profesor del guía de la escalada representa el entusiasmo para alcanzar una meta (véase figura 5) y tiene un significado situado-social por lo que representa el trabajo en equipo (Cassel y Vincent, 2011).

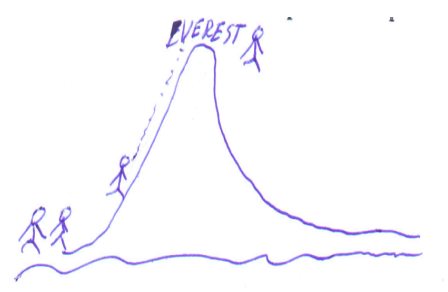

Figura 5. Dibujos del profesor y los estudiantes escalando una montaña

Perseverancia: El profesor se identifica con la metáfora cognitiva-constructivista del sembrador, asociada a la de las semillas que germinan para el alumnado (Seung, Park y Narayan, 2011). El profesor siembra la semilla y tiene que perseverar regando, abonando y cuidando las plantas en su crecimiento (véase figura 6). 


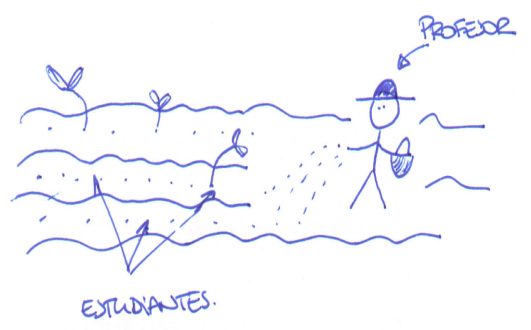

Figura 6. Dibujos del profesor como sembrador y de los estudiantes como semillas y plantas

También significa perseverancia la metáfora de la hormiga (véase figura 7), que en el dibujo se muestra escribiendo en la pizarra sin participación de los alumnos, por lo que tiene connotaciones transmisivas.

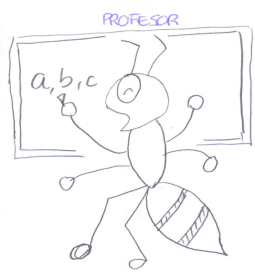

Figura 7. Dibujo del profesor como una hormiga

Estímulo/Motivación: La metáfora del director de orquesta representa estas emociones (véase figura 8). Esta metáfora ha sido habitualmente catalogada como situada, porque el director dirige a un colectivo, pero cada alumno toca un instrumento diferente (Ben-Peretz et al., 2003; Levy et al., 2007).

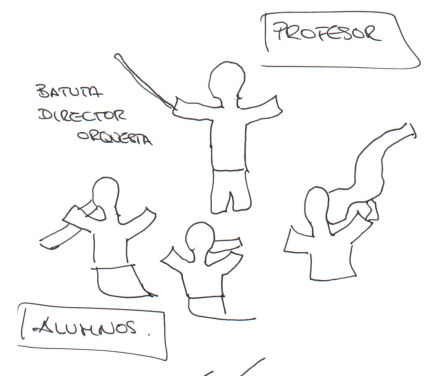

Figura 8. Dibujos del profesor como director de orquesta y de los alumnos como músicos

Transformación: Para el alumnado la metáfora cognitiva-constructivista de la oruga que se transforma en mariposa (véase figura 9), similar a las referenciadas para los estudiantes por Seung el at. (2011), refleja organismos que cambian y se desarrollan y que inciden más en el proceso del aprendizaje que en el producto final. 


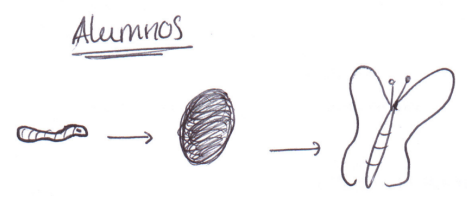

Figura 9. Dibujo para la metáfora de los alumnos, de la oruga que se transforma en mariposa

Protección/seguridad: Para el profesor la metáfora del perro guía como lazarillo puede clasificarse entre situada, por ser guía, y cognitiva-constructivista, por ser una ayuda para el ciego (alumno), que sin embargo toma sus propias decisiones (véase figura 10).

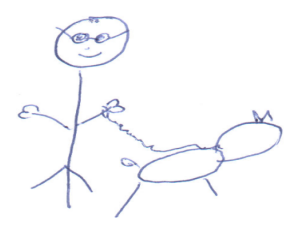

Figura 10. Dibujo del profesor como perro lazarillo.

c) Metáforas que reflejan emociones primarias negativas. Para el profesor hemos identificado la emoción del miedo en la metáfora del cordero entre lobos. No se han identificado emociones primarias negativas para el alumnado.

d) Metáforas que reflejan emociones sociales negativas. Intimidación: Las metáfora de la mirada asesina (pretest) o de la mirada del lobo (postest) del profesor (véase figura 11). Estas metáforas tienen un alto grado de agresividad y son coherentes con las metáforas expresadas por este participante para los alumnos: bichos que no paran de moverse.

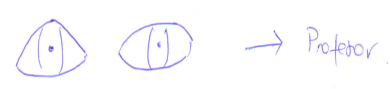

Figura 11. Dibujos de la mirada asesina del profesor

Inseguridad: esta emoción se asocia a la metáfora de la montaña rusa para el alumnado (véase figura 12), debido a los cambios de carácter que viven los adolescentes en su vida diaria. 


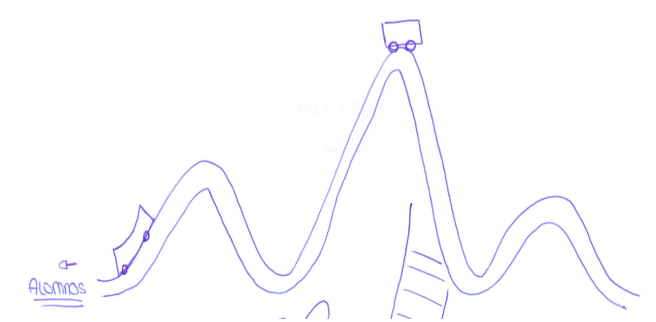

Figura 12. Dibujos de la montaña rusa para el alumnado

Sumisión: la metáfora de la marioneta con el profesor moviendo los hilos de los alumnos-muñecos (véase figura 13) se ha identificado en los estudios previos como fuertemente conductista (BenPeretz et al., 2003; Saban, 2010). También refleja sumisión para el alumnado la metáfora de las ovejas.

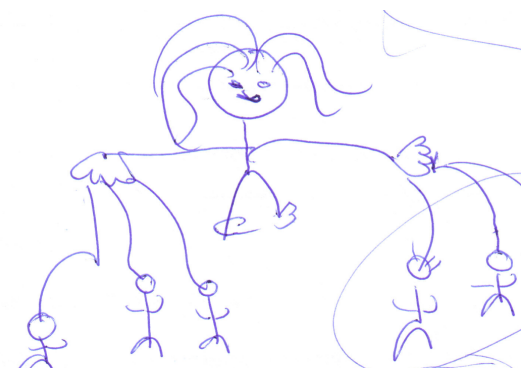

Figura 13. Dibujos para las metáforas de la marioneta

\section{Conclusiones e implicaciones didácticas}

a) Todos los participantes han sido capaces de conceptualizar en forma de metáforas los roles del profesor y del alumnado. Un resultado coincidente con anteriores estudios (Mahlios et al., 2010; Mellado, Bermejo y Mellado, 2012; Mellado et al., 2016a) es que las metáforas no están asociadas a contenidos concretos, sino que expresan una visión general de la enseñanza y del rol del profesor y del alumnado, formadas de sus propias experiencias como alumnos o docentes.

Antes de las prácticas el mayor porcentaje de metáforas para el profesor son las transmisivas-conductistas, resultado que coincide con otros estudios con futuros profesores de carreras universitarias de Ciencias, Economía o Tecnología, cuyas titulaciones universitarias, antes de realizar el Máster, se centran en el conocimiento del contenido y no tienen orientación hacia la docencia (Akcay, 2016; Mellado et al., 2012; 2016a). Si como indican Tobin et al. (1994) los futuros profesores comienzan el Máster asumiendo los roles de los profesores que han tenido en sus propios estudios, el mayor número de metáforas conductistas-transmisivas en el pretest puede ser indicativo de que los modelos conductistas-transmisivos han predominado en su formación previa. En cambio en la Licenciatura de Psicopedagogía o en la especialidad de Orientación Educativa del MFPES, en las que los participantes llevan varios años de estudios relacionados con la educación, el mayor número de metáforas son cognitivas-constructivistas, incluso en el pretest (Mellado et al., 2016b). Después de las prácticas, el mayor porcentaje de metáforas en la muestra de Economía son cognitivas-constructivistas y situadas, destacando la disminución de las transmisivas-conductistas y el gran aumento de las situadas-sociales, metáforas de orientación social que contrastan con el alto 
grado de individualismo en su ejercicio diario señalado por Carroza y Travé (2012) para el profesorado de Economía de secundaria. En consonancia con otros estudios (Kavanov, 2016; Seung et al., 2011), en la muestra de Economía se producen cambios significativos en el postest hacia orientaciones más centradas en el alumnado.

El mayor porcentaje de metáforas para el alumnado en el pretest son las autorreferenciadas, seguidas de las cognitivas-constructivistas y transmisivas-conductistas. Después de las prácticas aumentan las metáforas transmisivas-conductistas y cognitivas-constructivistas y hay una fuerte disminución de las autorreferenciadas, aunque existe poca coherencia con las categorías mostradas para el profesor.

b) En cuanto a la evolución de los modelos didácticos asociados a las metáforas, destacamos que ocho participantes de 14 (el 57.1\%) no cambian sus modelos, ni para el profesor ni para el alumnado, lo cual indica la gran estabilidad de los modelos asociados a las metáforas, que están ya muy asentados al comienzo del máster. Para el alumnado los modelos son aún más estables, ya que el 78.5\% no los cambia después de las prácticas. Sin embargo, también se produce progresión en algunos participantes, especialmente en las metáforas sobre el profesor, por lo que consideramos que la intervención y la reflexión sobre las metáforas tienen potencialidad para hacerlos conscientes de sus concepciones y roles docentes y para iniciar cambios en los mismos hacia modelos didácticos más centrados en el alumnado.

c) A diferencia de las metáforas generales, en las emocionales el mayor porcentaje sobre el rol del profesor corresponde a la categoría cognitiva/constructivista, aumentando después de las prácticas las metáforas de las categorías cognitiva/constructivista y situada-social. Para el alumnado el mayor porcentaje de metáforas emocionales concierne a las categorías cognitiva/constructivista y autorreferenciada. Después de las prácticas aumentan los porcentajes de las metáforas emocionales en las categorías conductista/transmisiva y situada-social y disminuyen en las otras dos.

d) En cuanto a las emociones asociadas a las metáforas, señalamos que tanto las metáforas emocionales sobre el profesor como las del alumnado reflejan en su mayoría emociones sociales positivas. Para el profesor destacamos que después de las prácticas aumenta la proporción de las emociones sociales y positivas, sobre las primarias y negativas, respectivamente. Para el alumnado después de las prácticas aumenta la proporción de emociones sociales, respecto a las primarias, pero prácticamente permanecen en la misma proporción las emociones positivas y negativas.

Para el rol del profesor destaca la emoción positiva primaria de la felicidad, aunque la mayor parte son emociones sociales positivas como entusiasmo, afecto, estímulo/motivación, protección, paciencia, competencia, adaptación, diversión, compromiso, afinidad, valor, respeto o esfuerzo. Para el profesor existe la emoción negativa primaria del miedo, aunque hay más emociones negativas sociales como intimidación control o inflexibilidad. Para el alumnado, el mayor número son emociones sociales, tanto positivas (entusiasmo, estímulo, ilusión, seguridad, transformación, adaptación o vivacidad), como negativas (inseguridad, desprecio, apatía, desorientación o sumisión).

Con relación a las implicaciones didácticas, consideramos que para que se produzca una mayor progresión en los modelos haría falta un trabajo común en las diversas asignaturas del Máster, que deberían orientarse hacia la profesionalización docente y no a la ampliación de los contenidos de las carreras. También debería existir una mayor relación y coherencia entre los módulos teóricos del Máster y las prácticas de enseñanza, las cuales no deben ser una mera aplicación de los módulos anteriores, sino una fuente de conocimiento profesional, que debe estimular y retroalimentar la reflexión metacognitiva del Máster, para lo cual las prácticas finalistas, no parece la mejor opción.

En futuras investigaciones sería importante analizar los factores que estimulan o dificultan los 
cambios en las metáforas. También habría que profundizar en la intervención, así como analizar no sólo lo que el profesor dice, sino lo que el profesor hace en el aula, para contrastar las metáforas y modelos declarados con los observados en la práctica del aula.

\section{Referencias}

Akcay, S. (2016). An analysis of teachers' perceptions through metaphors: Prospective Turkish teachers of science, math and social science in secondary education. Educational Research and Reviews, 11(24), 2167-2176.

Ben-Peretz, M., Mendelson, N. \& Kron, F.W. (2003). How teachers in different educational contexts view their roles. Teaching and Teacher Education, 19(2), 277-290.

Borrachero, A.B., Brígido, M., Mellado, L., Costillo. E., \& Mellado, V. (2014). Emotions in prospective secondary teachers when teaching science content, distinguishing by gender. Research in Science \& Technological Education, 32(2), 182-215.

Buaraphan, K. (2011). Metaphorical roots of beliefs about teaching and learning science and their modifications in the standard based science teacher preparation programme. International Journal of Science Education, 33(11), 1571-1595.

Cañal, P.; Travé, G. \& Pozuelos, F. J. (2011). Análisis de obstáculos y dificultades de profesores y estudiantes en la utilización de enfoques de investigación escolar. Investigación en la Escuela, 73, $5-26$.

Cañas, F. \& de la Montaña, J.L. (2012). Reflexiones sobre el desarrollo profesional del profesorado de Economía. Tejuelo, 15, 101-118.

Carroza, M. \& Travé, G. (2012). Análisis de las concepciones del profesorado de economía sobre satisfacción docente y desarrollo profesional. Investigación en la Escuela, 76, 23-36.

Cassel, D. \& Vincent, D. (2011). Metaphors reveal preservice elementary teachers' views of mathematics and science teaching. School Science and Mathematics, 111(7), 319-324.

Consejo Nacional de Educación Económica [Joint Council on Economic Education] (1985). Economic education for future elementary and secondary teachers: basic recommendations. New York.

Damasio, A. (2010). Y el cerebro creó al hombre. Barcelona: Editorial Destino.

Dodd, S.D. (2002). Metaphors and meaning: A grounded cultural model of US entrepreneurship. Journal of Business Venturing, 17(5), 519-535.

Estepa, J. \& Cuenca, J.M. (2007). Las concepciones de los docentes y el desarrollo profesional: dos estudios desde la formación inicial en Ciencias Sociales. Investigación en la Escuela, 61, 85-98.

Fukuda, K. (2009). A comparative study of metaphors representing the US and Japanese economies. Journal of Pragmatics, 41(9), 1693-1702.

Gutiérrez, P. \& Luengo, R. (2003). Orientación vocacional y género. Campo Abierto, 23(1), 85-98.

Herrera-Soler, H. \& White, M. (2012). Metaphors and Mills. Berlin: De Gruyter Mouton.

Kavanoz, S. (2016). Unveiling Pre-service Language Teachers' Conceptualizations of Teachers of English through Metaphors. Journal of Education and Training Studies, 4(10), 17-32.

Lakoff, G. \& Johnson, M. (1986). Metáforas de la vida cotidiana. Madrid: Cátedra.

Leavy, A.M., McSorley, F.A. \& Boté, L.A. (2007). An examination of what metaphor construction reveals about the evolution of preservice teachers' beliefs about teaching and learning. Teaching and Teacher Education, 23, 1217-1233.

Madhavaram, S. \& McDonald, R.E. (2010). Knowledge-based sales management strategy and the grafting metaphor: Implications for theory and practice. Industrial Marketing Management, 39(7), 1078-1087. 
Mahlios, M., Massengill-Shaw, D. \& Barry, A. (2010). Making sense of teaching through metaphors: a review across three studies. Teachers and Teaching: Theory and Practice, 16(1), 49-71.

Markic, S. \& Eilks, I. (2012). A comparison of student teachers' beliefs from four different science teaching domains using a mixed.method design. International Journal of Science Education, 34(4), 589-608.

Martínez, M.A., Sauleda, N. \& Huber, G.H. (2001). Metaphors as blueprints of thinking about teaching and learning. Teaching and Teacher Education, 17(8), 965-977.

McCorkle, S. \& Gayle, B.M. (2003). Conflict management metaphors: assessing everyday problem communication. The Social Science Journal, 40(1), 137-142.

Mellado, L., Bermejo, M.L. \& Mellado, V. (2012). Personal metaphors of prospective secondary economics and science teachers. Asia Pacific Journal of Teacher Education, 40(4), 395-408.

Mellado, L., Luengo, M.R., de la Montaña, J.L. \& Bermejo, M.L. (2015). Emotions in Personal Metaphors of Prospective Secondary Economics, Science, and Psychopædagogy Teachers. In A. Tavidze (ed.), Progress in Economics Research, vol. 30 (pp.27-46). New York: Nova Science Publishers.

Mellado, L., de la Montaña, J.L., Luengo, M.R. \& Bermejo, M.L. (2016a). Evolución de los modelos docentes personales de futuros profesores de Economía de Secundaria, a través de la metáfora. Didáctica de las Ciencias Experimentales y Sociales, 30, 23-44.

Mellado, L., Luengo, M.R., de la Montaña, J.L. \& Bermejo, M.L. (2016b). Las metáforas emocionales de profesores en formación de Infantil, Primaria y Psicopedagogía. Enseñanza \& Teaching, 34(1), 179-195.

Mellado, L., Parte, L. Garvey, A.M. (2016). Future managers' personal metaphors: an exploratory study with MBA students. Progress in Economics Research. New York: Nova Science Publishers. (en prensa).

Mellado, V., Borrachero, A. B., Brígido, M., Melo, L.V., Dávila, M.A., Cañada, F., Conde, M.C., Costillo, E., Cubero, J., Esteban, R., Martínez, G., Ruiz, C., Sánchez, J., Garritz, A., Mellado, L., Vázquez, B., Jiménez, R. \& Bermejo, M.L. (2014). Las Emociones en la Enseñanza de las Ciencias. Enseñanza de las Ciencias, 32(3), 11-36.

Molina, J.A. \& Travé, G. (2014). Concepciones y prácticas del profesorado de economía sobre el uso de materiales curriculares: un análisis exploratorio. Enseñanza de las Ciencias Sociales, 13, 71-83.

Morgan, G. (1980). Paradigm, metaphors, and puzzle solving in organization theory. Adminstrative Science Quarterly, 25(4), 605-622.

National Research Council (1996). The National Science Education Standards. Washington.

Porlán, R. \& Rivero, A. (1998). El conocimiento de los profesores. Una propuesta formativa en el área de ciencias. Sevilla: Díada.

Rebollo, M.A., Jiménez, R., Sabuco, A. \& Vega, L. (2013). Metáforas que usan las profesoras en los procesos de cambio educativo con perspectiva de género. Profesorado: Revista de currículum y formación del profesorado, 17(1), 43-56.

Saban, A. (2010). Prospective teachers' metaphorical conceptualizations of learner. Teaching and Teacher Education, 26(2), 290-305.

Salemi, M.K. (2003). A model teacher-education program for economics. The American Economic Review, 93(2), 455-459.

Salemi, M.K., Siegfried, J.J., Sosin, K., Walstad, W.B. \& Watts, M. (2001). Research in economic education: Five new initiatives. The American Economic Review, 91 (2), 440-445.

Seung, E., Park, S. \& Narayan, R. (2011). Exploring elementary pre-service teachers' beliefs about science teaching and learning as revealed in their metaphor writing. Journal of Science Education and Technology, 20(6), 703-714 
Shaw, D.M., Barry, A. \& Mahlios, M. (2008). Preservice teachers' metaphors of teaching in relation to literacy beliefs. Teachers and Teaching: Theory and Practice, 14(1), 35-50.

Teichert, T., von Wartburg, I. \& Braterman, R. (2006). Tacit meaning in disguise: Hidden metaphors in new product development and market making. Business Horizons, 49(6), 451-461.

Tobin, K., Tippins, D.J. \& Gallard, A.J. (1994). Research on instructional strategies for teaching science. In D. Gabel (ed.), Handbook of Research on Science Teaching and Learning (pp. 3-44). New York: MacMillan.

Travé, G. (1998). La investigación en didáctica de las ciencias sociales. Huelva: Publicaciones de la Universidad de Huelva.

Travé, G. \& Pozuelos F. (2008). Enseñar economía mediante estrategias de investigación escolar. Estudio de caso sobre las concepciones y prácticas del profesorado. Enseñanza de las Ciencias Sociales, 7, 109-120.

Walters, M., \& Young, J. J. (2008). Metaphors and accounting for stock options. Critical Perspectives on Accounting, 19(5), 805-833.

\section{Información sobre los autores}

Autora: Lucía Mellado Bermejo

Institución: Departamento de Economía de la Empresa y Contabilidad. Facultad de Ciencias Económicas y Empresariales, UNED, Madrid

E-mail:1mellado@cee.uned.es

Información biográfica: Licenciada en Economía y Derecho y Dra. en Economía, es Profesora Ayudante Doctora en la UNED. Sus líneas de investigación son la normalización contable y las metáforas y las emociones en la formación del profesorado. Ha publicado libros en editoriales internacionales, así como artículos en revistas nacionales e internacionales como Asia Pacific Journal of Teacher Education, Research in Science \& Technological Education, Revista Mexicana de Investigación Educativa 0 Didáctica de las Ciencias Experimentales y Sociales, entre otras.

Autor: Juan Luis de la Montaña Conchiña

Institución: Departamento de Didáctica de las Ciencias Sociales y de las Lenguas y Literaturas. Facultad de Educación, Universidad de Extremadura

E-mail: jmoncon@unex.es

Información biográfica: Licenciado y Dr. en Historia, es Profesor Contratado Doctor de Didáctica de las Ciencias Sociales. Sus líneas de investigación son el dominio social y afectivo en la didáctica de las ciencias sociales. Ha publicado libros en editoriales nacionales y artículos en revistas nacionales e internacionales como Didáctica de las Ciencias Experimentales y Sociales, Journal of Education Research, Revista Mexicana de Investigación Educativa o Enseñanza and Teaching, entre otras.

Autora: María Rosa Luengo González

Institución: Departamento de Didáctica de las Ciencias Sociales y de las Lenguas y Literaturas. Facultad de Educación, Universidad de Extremadura

E-mail: rosaluen@unex.es

Información biográfica: Licenciada Filosofía y Letras (Filología) y Dra. en Educación, es Profesora Titular de Didáctica de la Lengua y la Literatura. Sus líneas de investigación son los estudios de 
género en la educación y el dominio social y afectivo en la didáctica de las ciencias sociales. Ha publicado libros, capítulos de libros y artículos en revistas nacionales e internacionales como Feministische studien, BROCAR, Revista de Literatura, Revista Electrónica Interuniversitaria de Formación del Profesorado, Revista Mexicana de Investigación Educativa o Enseñanza and Teaching, entre otras.

Autora: María Luisa Bermejo García

Institución: Departamento de Psicología y Antropología. Facultad de Educación, Universidad de Extremadura

E-mail: mbermejo@unex.es

Información biográfica: Licenciada en Filosofía y Letras (Psicología) y Doctora en Psicología, es Profesora Titular de Psicología Evolutiva y de la Educación. Sus líneas de investigación son las emociones en la formación del profesorado y la atención a la diversidad. Ha publicado libros, capítulos de libros y artículos en revistas nacionales e internacionales internacionales como European Journal of Teacher Education, Asia Pacific Journal of Teacher Education, International Journal of Science and Mathematics Education, Science \& Education o Journal of Science Education for Students with Disabilities, entre otras. 


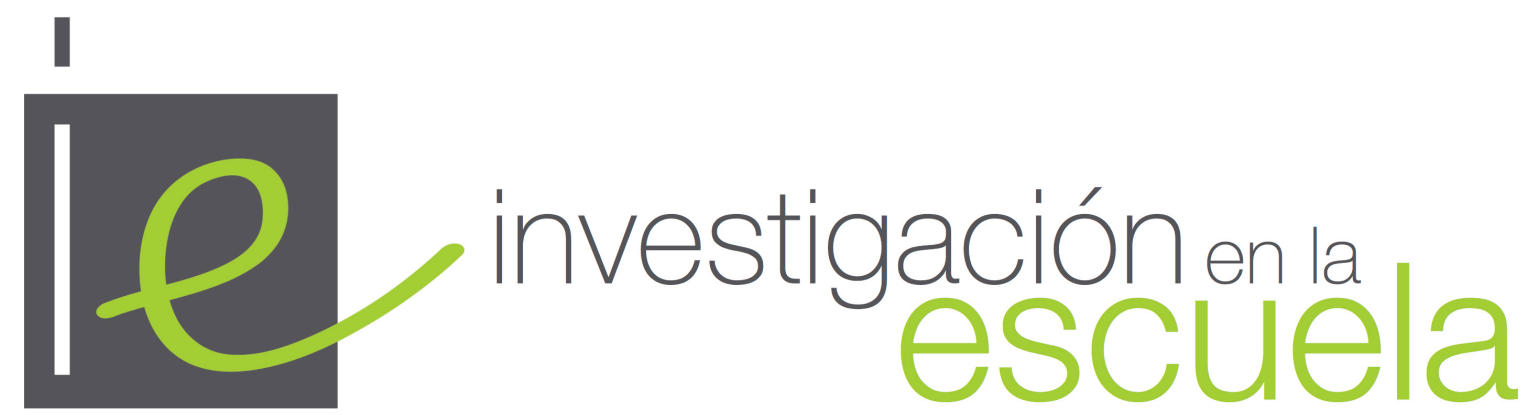

Revista internacional de investigación e innovación educativa

Número 91

06 de julio de 2017

ISSN 2443-9991

\section{(c)}

SORERIIGHISRESERVEDLOS/as lectores/as pueden copiar, mostrar, y distribuir este artículo, siempre y cuando se de crédito y atribución al autor/es y a Investigación en la Escuela, se distribuya con propósitos no-comerciales, no se altere o transforme el trabajo original. Más detalles de la licencia de CreativeCommons se encuentran en http://creativecommons.org/licenses/by-nc-sa/3.0 Cualquier otro uso debe ser aprobado en conjunto por el autor/es, o Investigación en la Escuela.

Uิ investigacion-en-la-escuela

Contribuya con comentarios y sugerencias en la web de la revista. Por errores y sugerencias contacte a investigacionescuela@ddcc.uhu.es 


\section{Investigación en la escuela}

Consejo de dirección: Ana Rivero García (Universidad de Sevilla), Nicolás de Alba Fernández(Universidad de Sevilla), Pedro Cañal de León (Universidad de Sevilla), Francisco F. García Pérez (Universidad de Sevilla), Gabriel Travé González, (Universidad de Huelva), Francisco F. Pozuelos Estrada (Universidad de Huelva)

Dirección: Ana Rivero García y Nicolás de Alba Fernández Secretaría de edición: Elisa Navarro Medina

\section{Consejo editorial}

José Félix Angulo Rasco. Universidad de Cádiz Rosa Ma Ávila Ruiz. Universidad de Sevilla Pilar AzcárateGoded. Universidad de Cádiz Juan Bautista Martínez Rodríguez. Universidad de Granada

Nieves Blanco García. Universidad de Málaga Fernando Barragán Medero. Universidad de La Laguna José Carrillo Yáñez. Universidad de Huelva José Contreras Domingo. Universidad de Barcelona. Luis C. Contreras González. Universidad de Huelva Ana $\mathbf{M}^{\mathbf{a}}$ Criado García-Legaz. Universidad de Sevilla Rosario Cubero Pérez. Universidad de Sevilla José $\mathbf{M}^{\mathbf{a}}$ Cuenca López. Universidad de Huelva Jesús Estepa Giménez. Universidad de Huelva Rafael Feito Alonso. Universidad Complutense (Madrid)

Francisco José García Gallardo. Universidad de Huelva

Soledad García Gómez. Universidad de Sevilla J. Eduardo García Díaz. Universidad de Sevilla
Fernando Hernández Hernández. Universidad de Barcelona

Salvador Llinares Ciscar. Universidad de Alicante Alfonso Luque Lozano. Universidad de Sevilla Rosa Martín del Pozo. Universidad Complutense (Madrid)

José Martín Toscano. IES Fernando Herrera (Sevilla) Jaume Martínez Bonafé. Universidad de Valencia F. Javier Merchán Iglesias. Universidad de Sevilla Emilia Moreno Sánchez. Universidad de Huelva.Rosario Ortega Ruiz. Universidad de Córdoba

Antonio de Pro Bueno. Universidad de Murcia Fco. de Paula Rodríguez Miranda. Universidad de Huelva

Pedro Sáenz-López Buñuel. Universidad de Huelva Antoni Santisteban Fernández. Universidad Autónoma (Barcelona) Emilio Solís Ramírez. Catedrático de IES. $\mathbf{M}^{\mathbf{a}}$ Victoria Sánchez García. Universidad de Sevilla. Magdalena Suárez Ortega. Universidad de Sevilla

\section{Consejo asesor}

Manuel Área Moreira. Universidad de La Laguna

Jaume Carbonell. Director Cuadernos de Pedagogía. Barcelona

César Coll. Universidad de Barcelona

Christopher Day. Universidad de Nothingham. U.K.

Juan Delval. Universidad Nacional de Educación a Distancia

John Elliott. Universidad de East Anglia. Norwich. U.K.

José Gimeno Sacristán. Universidad de Valencia

André Giordan. Universidad de Paris VII y Ginebra

Francisco Imbernón. Universidad de Barcelona

Ángel Pérez Gómez. Universidad de Málaga

Rafael Porlán Ariza. Universidad de Sevilla

Francesco Tonucci. Instituto de Pedagogía del C.N.R. Roma

Jurjo Torres Santomé. Universidad de A Coruña 
